\section{Mental Health among Syrian Immigrants in Iraq}

\author{
Firouzeh Sepehrianazar', \\ Sara Qader Tilee ${ }^{1}$ and \\ Margarida Gaspar de \\ Matos ${ }^{2 *}$ \\ 1 Urmia University, Urmia, Iran \\ 2 Faculty of Human Medicine, Institute of \\ Environmental Health, University of Lisbon \\ / FMH-ISAMB-FCT, Portugal
}

Background: The migration of Syrian citizens to many parts of the world began
after civil war in Syria. Many migrants lost their homes, friends and relatives as a result of the civil war. Millions of Syrians have been refugees to European countries and especially to neighboring countries such as Libya, Turkey and Iraq. They have experienced housing, safety and cultural gap problems, which may put their mental health at risk. Many present signs of physical and mental illness, such as an insomnia, a weakened immune system, drug addiction, self-harm, and aggression, making their social adjustment more and more difficult. Despite facing the same conditions, other immigrants succeed to adjust with the new environment. It is necessary to understand factors that may optimize immigrants' social adjustment.

Objectives: The aim of the present study is to evaluate to what extend some characteristics of Syrian immigrants in Iraq, such as cultural intelligence, emotional intelligence are related to their social adjustment, and the role of mental health upon that relationship.

Methods: Participants are Syrian immigrants who live in Erbil - Iraq. Data collection was held in 2017 . Were randomly included 288 subjects ( $50 \%$ female). All participants completed 4 questionnaires translated from Persian version to Arabic language: Cultural and Emotional Intelligence Questionnaires $(\mathrm{Cl}$ and $\mathrm{EI}), \mathrm{a}$ Social Adjustment scale (SA) and a Mental Health Questionnaire (MH).

Results: This study presents an empirical study into the mental health of immigrants. More specific, based on collected questionnaire data an explanatory model for mental health and social adjustment is established on the basis of emotional- and cultural intelligence. A better mental health is associated with better social adjustment. Emotional and cultural intelligence are significantly and positively and significantly and positively associated to social adjustment. Cultural intelligence is significantly and positively related to mental health, while emotional intelligence has no significant relation with mental health, when simultaneous effects are analyzed.

Conclusions: The present results suggest that mental health may have an intermediate role in the relationship between cultural intelligence and social adjustment, but no intermediate role in the relationship between emotional intelligence and social adjustment. Results convey an important message to public policies, highlighting the importance of preserving both cultural and emotional intelligence among Syrian immigrant in Iraq, as a measure to assure their mental health and social adjustment.

Keywords: Immigrants; Cultural intelligence; Emotional intelligence; Mental health; Social adjustment
*Corresponding author: Margarida Gaspar de Matos

झ mmatos@fmh.ulisboa.pt; margarida.gaspardematos@gmail.com

Professor, Faculty of Human Medicine, Institute of Environmental Health, WFH/University of Lisbon/ FMH-ISAMB-FCT, Estrada da Costa 1495-688 Cruz Quebrada, Portugal.

Tel: + 351-21-414-91-52

Citation: Sepehrianazar F, Tilee SQ, Matos MGD (2019) Mental Health among Syrian Immigrants in Iraq. Acta Psychopathol Vol.5 No.2:1 


\section{Background}

Each year millions of people change their life worlds, either against their will or voluntarily, and nowadays, due to faster and more sophisticated communication tools and conditions, the world witnesses the increasing rise in immigration [1]. Immigration is one of the social behaviors issues [2] that cause changes in population structure, cultural and scientific transfer, and all types of voluntary or compulsory immigration face different challenges in a cross-cultural environment, while experiencing the change from a familiar environment to an unfamiliar [3].

The migration of Syrian citizens to many parts of the world began after civil war in Syria. Migrants are in general citizens who lost their homes, relatives and friends, as a result of the civil war. Millions of Syrians have been refugees to European countries and especially to neighboring countries such as Libya, Turkey and Iraq. Most of Syria Refugees to Iraq who are Kurdish, live in Iraqi Kurdistan, Erbil [4]. They have experienced s problems, such as living in tents, limited facilities and cultural differences. There are signs of physical and mental illness among them, such as an insomnia, a weakened immune system, drug addiction, self-harm, and aggression, making their adjustment with the environment more and more difficult [4]. Despite facing the same conditions, some immigrants succeed to adjust with the new environment. It is necessary to understand factors that may optimize immigrants' social adjustment.

\section{Theoretical Background}

Adjustment refers to all strategies that a person uses to handle life stressful situations [5]. Social adjustment differs from society to society, according to the culture and the beliefs of the community. According George, Thamson, Chaze \& Guruge [6] mental health is pivotal to holistic health and wellbeing. Mental health is a dynamic state of mental satisfaction and a feeling that one can cope with the community and enjoy personal situations and social characteristics $[7,8]$, quoting the humanistic psychologists, believe that the love, approval and positive nonconditional attention of the child is essential for the development of a person's mental health.

Cultural Intelligence, which refers to the ability to effectively interact with cultures, is a concept that was first introduced by Early \& Ang [9] and comes into play in the analysis and adaptation to different cultures. They define cultural intelligence as the person's ability to successfully adapt to new cultural environments, usually different from one's own cultural context. The results of Guomundsottir's [10] study showed that there is a significant correlation between a greater general adjustment and greater meta-cognitive and motivational cultural intelligence. Cultural intelligence enables the individual to recognize cultural differences and to behave properly in different cultural environments [11]. Early \& Mosakowski [12] presented cultural intelligence has having three dimensions: cognitive, physical / behavioral, and motivational, and Thomas [13] considered three components: cultural knowledge, cultural attention and cultural skills. Harris, Lievens, [14] associated this concept with other types of intelligence, including emotional and social intelligence. Tabatabaeimozabadi [15] claimed that there is a significant correlation between Emotional Intelligence \& Cultural Intelligence.

Emotional intelligence is one of the factors that affect cultural intelligence, although Ang, Van Dyne, Koh, Templer, Tay \& Chandrasekar [16] argued that cultural intelligence is conceptually and empirically different from emotional intelligence. Emotional intelligence is the appropriate processing of the data which has emotional load. Various scholars such as Bar-On [17], Sepehrianazar [18] and Goleman [19] have been working on conceptualizing emotional intelligence. Mayer, Salovey \& Caruso [20] believe that people with high emotional intelligence can control own emotions and others'. According to their theory, emotional intelligence consists of four different abilities: perception of emotion, emotional facilitation, understanding emotions, and management of emotions. Parsamehr \& Heddat [21] showed a direct prelationship pbetween pemotional pintelligence pand psocial padjustment among407 p pstudents pof pYazd pUniversity, Iran and, according to Sepehrianazar's study [22]. Emotional intelligence has significant effects on psychological health and on the individuals' behaviours.

The aim of the present study is to evaluate to what extend some characteristics of Syrian immigrants in Iraq, such as cultural intelligence, emotional intelligence are related to their social adjustment, and the role of mental health upon that relationship.

\section{Methods}

The aim of the present study is to evaluate to what extend some characteristics of immigrants, such as cultural intelligence, emotional intelligence and mental health, are related to their social adjustment in the host country and the role of mental health upon that relationship

A diagrammed model (Figure 1) was designed to propose a conceptual working model of the direct and indirect effects of the above-mentioned variables, the pattern(s) of relationship(s), and the effect of mental health.

\section{Participants}

Participants are Syrian immigrants who live in Erbil city of Iraq Country, in 2017. Were included 288 subjects (50\% female) randomly selected within the immigrants 'group. Participants age ranged from 20 to more than $40,(42 \% / n=121)$ had ages between 20 and $30 ; 40 \%(n=115)$ had ages between 31 and 40 years old, and $18 \%(n=52)$ were older than 40 (Mean age, 45.4 years old, SD, 3.77). $47.5 \%(n=116)$ of these participants had schooling up to secondary school, and $52.5 \%(n=128)$ had a university diploma.

\section{Data collection}

All participants volunteer to participate in the study and have completed four questionnaires. Data collection was anonymous. Study followed all usual ethics guidelines for research in humans. The study was carried on in the context of an academic research supervised by Urmia University and clearance of ethics issues was obtained at the University level. 


\section{Measures}

Besides demographics, participants answered the following questionnaires: 1) Cultural Intelligence Questionnaire (CIQ) of Earley \& Ang [9], (Lugo [23], translated to Persian language by Azemonyar Institute [24]); 2) Schutte Self-Report Emotional Intelligence Test (EIQ) (SSEIT) (Schutte, 1998 translated to Persian language by Hossaini [25]); 3) the Social Adjustment ScaleSelf-Report (SAS) of (Weissman \& Bothwell, 1999, translated to Persian language by Azemonyar Institute [24]), the Mental Health Questionnaire (GHQ) of Goldberg (Goldberg, Hillier [26], translated to Persian language by Nazifi, Mokarami, Akbaritabar, Faraji Kujerdi, Tabrizi \& Rahi [27]. Persian version of all tests were translated into Arabic and Kurdish language. All the translated instruments have followed international guidelines for accuracy (Questionnaires were translated from Persian version to Arabic language by two independent bilingual researchers and backtranslated for final check).

\section{Data analysis}

Descriptive and correlational analyses were conducted, followed by Structural Equation Modelling (SEM), to find the pattern of relationship between $\mathrm{El}$ and $\mathrm{Cl}$ with Social Adjustment in the host country while taking into account Mental Health. Significance level was set at $p<.05$

\section{Results}

Cultural Intelligence Questionnaire (CIQ) (Azemonyar Institute [24]), is a 20 items questionnaire ranging from min 20 to max 140. The goodness of fit indices for the CIQ on this study sample are adequate $X^{2}, \mathrm{df}=1.89$; SRMS $=0.06$; GFI=0.94 and $\mathrm{AGFI}=$ 0.91). Emotional Intelligence Questionnaire (EIQ) (Hossaini [25]), is a 33 items questionnaire ranging from $\min 33$ to $\max 165$. The goodness of fit indices for the EIQ on this study sample are adequate $X^{2}, \mathrm{df}=2.43$; $\mathrm{SRMS}=0.07 ; \mathrm{GFI}=0.92$ and $\mathrm{AGFI}=0.89$ ). Social Adjustment Scale (SAS) (Azemonyar Institute [24]) is a 54 items. For this study, students' questions were deleted and only 26 questions were used. To get the total test score, summing the scores of all items and dividing by the number of items actually scored. The goodness of fit indices for the SAS on this study sample are adequate $X^{2}, \mathrm{df}=2.81$; SRMS $=0.07 ; \mathrm{GFI}=0.91$ and $A G F I=0.88)$, and the Mental Health Questionnaire (MHQ/GHQ)
(Nazifi et al., [27]) is a 28 items questionnaire ranging from min 0 to max 84. The goodness of fit indices for the MHQ/ GHQ on this study sample are adequate $X^{2}, \mathrm{df}=2.39 ; \mathrm{SRMS}=0.07 ; \mathrm{GFI}=0.93$ and $\mathrm{AGFI}=0.90$ )

In what concerns Cultural Intelligence and Emotional Intelligence higher results correspond to more cultural or emotional intelligence respectively, while considering the Mental Health and Social Adjustment, higher scores correspond respectively to low or poor Mental Health and poor Social Adjustment.

\section{Bivariate analysis}

Descriptive analysis of the variables included in the study is presented (Table 1). Skewness and kurtosis indices suggested the normality of data distribution.

The correlations between research variables were calculated using Pearson's correlation coefficients (Table 2). All variables present significant correlations, emotional and cultural intelligence are significantly and positively correlated. Social adjustment is significantly and positively correlated to Mental Health.

According to the present results there is a negative and significant correlation among Emotional Intelligence and Cultural Intelligence and Mental Health, and a negative and significant correlation between Emotional Intelligence and Cultural Intelligence and Social Adjustment. Because lower scores on Mental Health and Social Adjustment correspond to respectively to a better Mental Health status and to a better Social Adjustment, this result must be read as an higher Emotional Intelligence and higher Cultural Intelligence being significantly associated to a better Mental Health, and an higher Cultural Intelligence and an higher Emotional Intelligence being significantly associated to a better Social Adjustment.

\section{Structural equations modeling}

A structural equations model was carried out in order to test simultaneous direct effects. In order to investigate Goodness of Fit index in the research model three indices were used one absolute, one comparative and another parsimonious (Kline [28]). In the current research, Nikoie's Goodness of Fit index (GFI), Nikoie's Adjusted Goodness of Fit Index (AGFI) and Standardized Root Mean Squared Residual (SRMR), and $\left(X^{2} / \mathrm{df}\right)$ square freedom

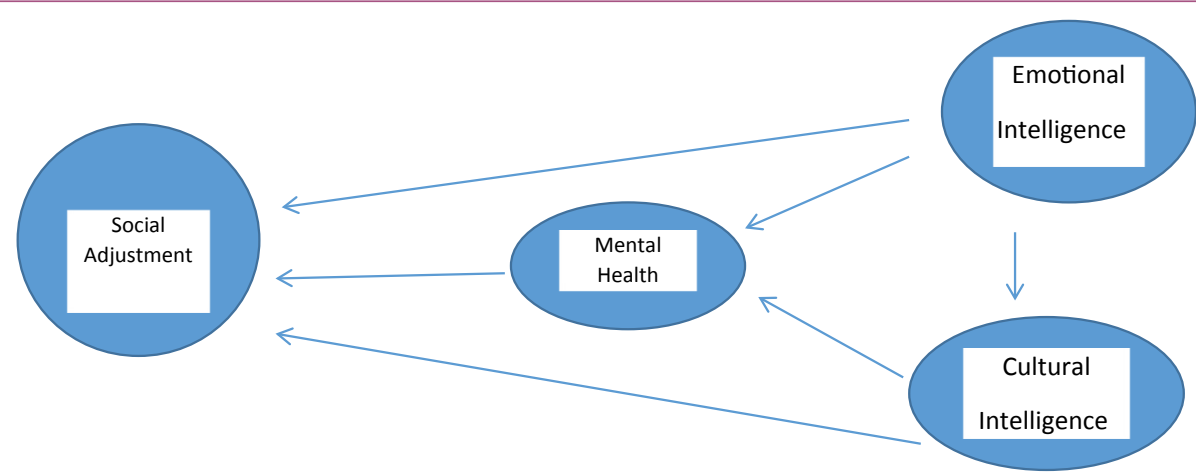

Figure 1 Conceptual working Model - relationship among the study variables. 
degree were calculated and are adequate $X^{2}, \mathrm{df}=2.06$; SRMS $=0.03 ; \mathrm{GFI}=0.91$ and $\mathrm{AGFI}=0.87$ ).

According to the present results, direct effect of Emotional Intelligence on Social Adjustment is significantly negative (-0.55). Mental Health direct effect on Social Adjustment is significantly positive (.15). Cultural Intelligence has a low, yet significant negative direct effect on social adjustment $(-0.08)$. Cultural Intelligence direct effect on Mental Health is significantly negative (-.35). Emotional Intelligence hasn't direct significant effect $(-0.08)$ on Mental Health. Emotion Intelligence directs effect on Cultural Intelligence is significantly positive [29]. As has been stated before, because lower scores on Mental Health and Social Adjustment correspond to respectively to a better Mental Health status and to a better Social Adjustment, results must be read as an higher Cultural Intelligence being significantly associated to a better Mental Health, and an higher Cultural Intelligence and an higher Emotional Intelligence being significantly associated to a better Social Adjustment (Table 3).

Emotional Intelligence, Cultural Intelligence and Mental Health explained $36 \%$ of Social Adjustment variance. Emotional Intelligence and Cultural Intelligence explained $14 \%$ of Mental Health variance. Emotional Intelligence explained $8 \%$ of Cultural Intelligence.

A diagrammed model (Figure 2) was designed present the direct and indirect effects of the above-mentioned variables, the pattern(s) of relationship(s), and the effect of mental health.

\section{Discussion}

At a bivariate level all variables presented significant correlations: emotional and cultural intelligence are significantly and positively correlated, and social adjustment is significantly and

Table 1: Summary of descriptive analysis of the variables included in the study.

\begin{tabular}{|c|c|c|c|c|c|c|}
\hline Variable & $\min$. & $\max$ & mean & $S D$ & skewness & kurtosis \\
\hline Emotional Intelligence (EI) & 38 & 149 & 102.27 & 22.60 & -0.56 & 0.19 \\
\hline Cultural Intelligence $(\mathrm{Cl})$ & 30 & 134 & 84.54 & 22.36 & -0.87 & -0.57 \\
\hline Mental health (MH) & 28 & 108 & 58.01 & 17.30 & 0.94 & 0.39 \\
\hline Social adjustment (SA) & 32 & 122 & 67.83 & 20.33 & 0.78 & 0.05 \\
\hline
\end{tabular}

Table 2: Summary of correlational analysis of the variables included in the study.

\begin{tabular}{|c|c|c|c|}
\hline Variable & EI & CI & MH \\
\hline Emotional Intelligence (EI) & 1 & & \\
\hline Cultural Intelligence (CI) & $0.30^{* *}$ & 1 & 1 \\
\hline Mental Health (MH) & $-0.17^{* *}$ & $-0.38^{* *}$ & $0.22^{* *}$ \\
\hline Social Adjustment (SA) & $-0.49^{* *}$ & $-0.15^{* *}$ & 1 \\
\hline
\end{tabular}

Table 3: Prediction of direct effect values.

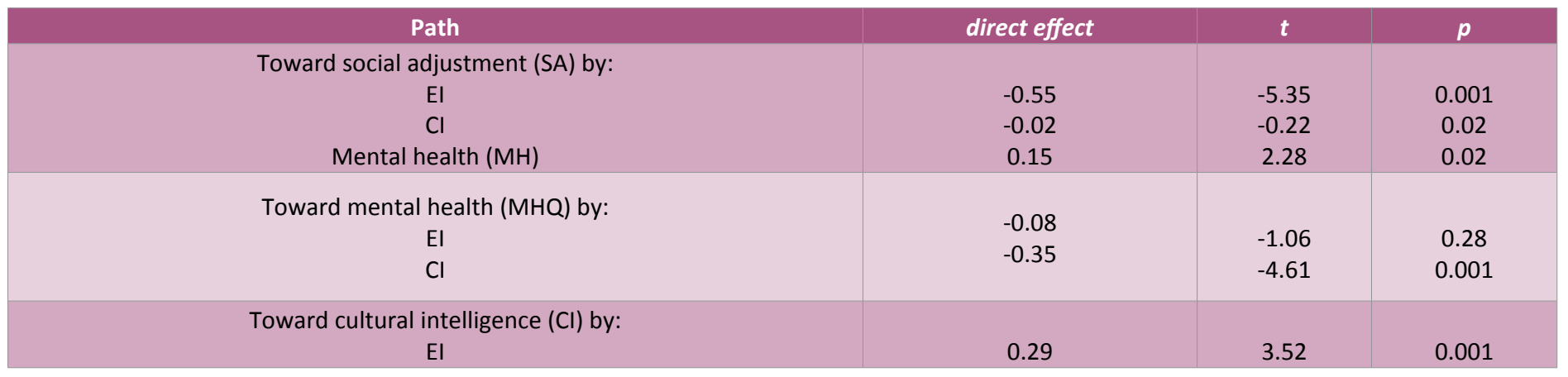

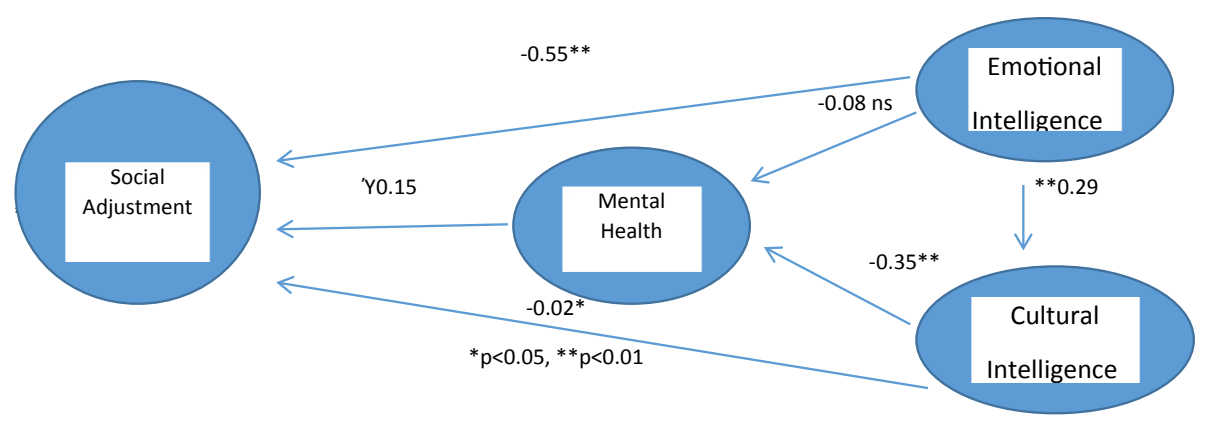

Figure 2 Structural Model-Direct effects of the relationship among study variables. 
positively correlated to mental health, Higher emotional and cultural intelligence are associated to a better mental health, and higher emotional and cultural intelligence are associated to a better social adjustment, as suggested by Early \& Ang [9], Guomundsottir [10] and Triandhs [11].

However other authors referred a multi-dimensional aspect of cultural adjustment (Early, \& Mosakowski, [12] and Thomas [13] that could not be analyzed in the present study.

At a multi-variated structural level, emotional intelligence has direct positive effect on cultural intelligence, as suggested by Tabatabaeimozabadi [15]. However as Ang et al. [16] argued that cultural intelligence may conceptually and empirically different from emotional intelligence, and this fact could better explain that, when analyzing simultaneous direct effects, emotional intelligence, hasn't a significant effect on mental health while cultural intelligence has. Also emotional intelligence has a direct effect on social adjustment much stronger than the direct effect of cultural intelligence, as suggested by Parsamehr \& Heddat [21]. In the case of emotional intelligence the results suggested that there is no mediational increase in the relation among emotional intelligence and social adjustment when mental health is considered.

Cultural intelligence has a much lower (yet significant) direct effect on social adjustment. Furthermore, in line with Ang's et al. [16] arguments, cultural intelligence has a significant direct effect on mental health (more cultural intelligence corresponding to a better mental health), opposite to the non-significant effect registered between mental health and emotional intelligence.

According to Mayer, Salovey \& Caruso [20] theory, emotional intelligence consists of four different abilities: perception of emotion, emotional facilitation, understanding emotions, and management of emotions. In the present study only a global measure was considered preventing detailed and partial sub scores to be analyzed.

Mental health has direct and significant effect social adjustment, meaning that a better situation regarding mental health is associated with better the social adjustment, as suggested before by Sepehrianazar [22].

The results of the present study highlight important implications for interventions, aiming at enhancing mental health and social adjustment of Syrian immigrants in Iraq. Emotional and cultural intelligence are significantly related, yet focusing different aspects that allow the different association they present with mental health. This fact is quite troublesome and may well be related to the poor study conditions, in very vulnerable conditions. It could also be better understood if subscales were considered in the related questionnaires. But it can also be the case that strong cultural bonds may be the key factor to increase resilience and lead to a better mental health, although not so efficient for social adjustment to new environments. On the opposite emotional intelligence, although not contributing to an enhanced mental health, could better suit for increased understanding of new environments thus promoting an increase social adjustment.

When analyzing simultaneous effects, a better mental health is associated with better social adjustment, and both emotional and cultural intelligence are significantly associated to social adjustment, but only cultural intelligence is significantly associated to mental health. The present results suggest that mental health may have an intermediate role in the relationship between cultural intelligence and social adjustment, but no intermediate role in the relationship between emotional intelligence with social adjustment.

\section{Limitations and Future Questions}

Future research must include qualitative methods in order to fully discuss the issues raised by this study with the immigrants, trying to work out their awareness and understanding of these results and their health implications (Matos [3]). Also it would be possible for a second analysis of this data in order to consider subscales, to be possible to be more analytical; identifying "which parts" of both emotional and cultural intelligence can be contributing to that twofold pattern. A comparative grade/schooling level, gender and age group analysis is also under preparation.

\section{Conclusions}

The authors aim with the publication of this work contributing to raise awareness and increase the well-being and social adjustment of Syrian immigrants, and in a very near future to be able to provide national governments with a few guidelines regarding their mental health and social adjustment promotion.

For the moment being the strongest conclusions are the relevance to focus on Syrian immigrants' mental health as a way to increase their social adjustment. A second issue is that cultural and emotional intelligence apparently have diverse (yet significant) effects on both mental health and social adjustment. Results convey an important message to public policies, highlighting the importance of preserving both cultural and emotional intelligence among Syrian immigrant in Iraq, as a measure to assure their mental health and social adjustment situations.

\section{Funding}

Matos, M. G. received a Sabbatical grant from the Foundation for Science and Technology (FCT) (SFRH/BSAB/135160/2017 INSERM/ Université Paul Sabatier/Toulouse/ France. 


\section{References}

1 Guedes F, Gaspar T, Tome G, Cerqueira A, Matos MG (2019) Os adolesentes portugeuses e a igualdade de oportunidades dos migrantes (Portuguese adolescents and equity among immigrants). J Child Adolesc Psychol 1: 95-106.

2 Zanjani H (2001) Immigration, Tehran: SAMT publisher.

3 Matos MG (2019) Dream teens: Navigating life like a protagonist, Saarbrucken: Lambert Academic Publishing.

4 Tilee SQ (2017) Mental health among syrian immigrants in Iraq, nonpublished dissertation presented at Urmia University, Urmia, Iran.

5 Sadock B, Sadock V, Kaplan H (2003) Kaplan \& sadock's synopsis of psychiatry: behavioral sciences/clinical psychiatry. Philadelphia, $\mathrm{Pa}$.: Lippincott Williams \& Wilkins.

6 George U, Thamson M, Chaze F, Guruge S (2015) Immigrant mental health. A public health issue: looking back and moving forward. Int J Environ Res Public Health 12: 13624-13648.

7 Milanifar B (2010) Mental health tehran: gomes. $11^{\text {th }}$ Edn.

8 Shfiabadi A, Naseri G (2017) Theories of counseling and psychotherapy. Tehran: university publication center (persian).

9 Early PC, Ang S (2003) Cultural intelligence: individual interactions across cultures. Stanford, CA: stanford university press.

10 Guomundsottir S (2015) Nordic expatriates in the US: the relationship between cultural intelligence and adjustment. Int J Intercult Relat 47: 175-186.

11 Triandhs HC (2006) Cultural intelligence in organizations. Group Organ Manag 31: 20-26.

12 Early PC, Mosakowski E (2004) Cultural intelligence. Harv Bus Rev 82: 139-146.

13 Thomas C (2006) Culture intelligence people skills for a global work place. Consulting to Management 16: 70-80.

14 Harris MM, Lievens F (2005) Selecting employees for global assignments: can assessment centers measure cultural intelligence? In A. Rahim \& R.T. Golembiewski (Eds.) Curr Top Manag 10: 221-240.

15 Tabatabaeimozabadi SM (2016) Study the role of emotional intelligence and cultural intelligence in the fields of organizational transformation tends. Journal of Innovation and Entrepreneurship 3: 105-116.
16 Ang S, Van Dyne L, Koh C, Templer KJ, Tay C, et al. (2007) Cultural intelligence: Its measurement and effects on cultural judgment and decision making, cultural adaptation and task performance. Management and Organization Review 3: 335-371.

17 Bar-On R (2000) Emotional and social intelligence: insights from the emotional quotient inventory. In: Bar-On R, Parker JDA, editors. The Handbook of Emotional Intelligence. San Francisco: Jossey Bass Pp: 363-388.

18 Sepehrianazar $F$ (2015) Emotional intelligence, from IQ to EQ and SQ. Iran: Jahad Daneshgahy Publications, $3^{\text {rd }}$ Edn, ISBN: 978-9646032-70-5.

19 Goleman D (1998) Working with emotional intelligence. New York: Bantam. Pp: 13-32.

20 Mayer JD, Salovey P, Caruso DR (2008) Emotional intelligence: new ability or eclectic traits?

21 Am Psychol 63: 503-517.

22 Parsamehr M, Heddat E (2017) The prelationship pbetween pemotional pintelligence pand psocial padjustment pof pstudents. J Soc Dev 11: 65-69.

23 Sepehrianazar F (2012) Emotional intelligence as a predictor for academic performance in university, J Educ Sci Psychol, Romania 2: 36-44.

24 Lugo M (2007) An examination of cultural and emotional intelligences in the development of global transformational leadership skills. Unpublished doctoral dissertation, Walden University.

25 Azemonyar Institute (2011) Social Adjustment scale (SAS).

26 Hossaini N (2009) Research questionnaire on psychology, counseling, education science and sociology. Tehran: Sokhan publisher.

27 Goldberg DP, Hillier VF (1979) A scaled version of the general health questionnaire. Psychol Med 9: 139-145.

28 Nazifi M, Mokarami H, Akbaritabar A, Faraji Kujerdi M, Tabrizi R, et al. (2013) Reliability, validity and factor structure of the persian translation of general health questionnaire (ghq-28) in hospitals of kerman university of medical sciences. J Fasa University of Med Sci 3: 336-342.

29 Kline RB (2011) Principles and practice of structural equation modeling. $2^{\text {nd }}$ Edn, New York: The Guilford Press. 